\title{
A Phase IIlb Open-Label, Single-Arm Study of Afatinib in EGFR TKI-Naïve Patients with EGFRm+ NSCLC: Final Analysis, with a Focus on Patients Enrolled at Sites in China
}

\author{
Hai-Yan Tu ${ }^{1}$. Jifeng Feng ${ }^{2} \cdot$ Meiqi Shi ${ }^{2}$ Jun Zhao ${ }^{3}$-Yuyan Wang ${ }^{3}$. Jianhua Chang ${ }^{4}$. Jialei Wang ${ }^{4} \cdot$ Ying Cheng $^{5}$. \\ Jing Zhu ${ }^{5}$. Eng-Huat Tan ${ }^{6} \cdot \mathrm{Kai} \mathrm{Li}^{7} \cdot$ Yiping Zhang ${ }^{8} \cdot$ Victor Lee $^{9,10}$. Cheng-Ta Yang ${ }^{11}$. Wu-Chou Su ${ }^{12}$. \\ David Chi-Leung Lam ${ }^{13}$. B. J. Srinivasa ${ }^{14}$. Senthil Rajappa ${ }^{15}$. Ching-Liang Ho ${ }^{16} \cdot$ Kwok Chi Lam $^{17}$. \\ $\mathrm{Yi} \mathrm{Hu}^{18}$. Shailesh Arjun Bondarde ${ }^{19}$. Xiaoqing $\mathrm{Liu}^{20}$ - Yahui Tian ${ }^{21}$. Zhiyi Xue ${ }^{21}$. Agnieszka Cseh ${ }^{22}$. \\ Dennis Chin-Lun Huang ${ }^{23} \cdot$ Caicun $\mathrm{Zhou}^{24}$. Yi-Long Wu ${ }^{1}$ (])
}

Accepted: 22 November 2021 / Published online: 12 January 2022

(c) The Author(s) 2021

\begin{abstract}
Background Afatinib has been shown as a suitable option for the treatment of epidermal growth factor receptor mutationpositive $(E G F R \mathrm{~m}+)$ non-small-cell lung cancer (NSCLC) in randomized controlled trials. However, patients treated in realworld clinical practice, including elderly patients, and those with brain metastases or poor Eastern Cooperative Oncology Group (ECOG) performance statuses, are often excluded from these studies.

Objective To report the final results, with a particular focus on patients enrolled in China, from a prospective phase IIIb, "near real-world" study of afatinib in tyrosine kinase inhibitor (TKI)-naïve Asian patients with EGFRm+ NSCLC.

Patients and Methods NCT01953913 was conducted at 34 centers across Asia. Entry criteria were broad to reflect real-world settings. Patients received afatinib $40 \mathrm{mg} /$ day until tumor progression, lack of clinical benefit, or poor tolerability. Assessments included safety, time to symptomatic progression (TTSP), and progression-free survival (PFS).

Results 541 patients were treated, of whom 412 were enrolled in China. Dose reductions were implemented in $28.7 \%$ of patients overall, and $17.7 \%$ of patients from China. Safety findings were consistent with phase III studies of afatinib. Median TTSP in all patients was 14.0 months (95\% CI 12.9-15.9), and median PFS was 12.1 months (95\% CI 11.0-13.6). Median TTSP (13.8 months, 95\% CI 12.7-16.1) and PFS (11.4 months, 95\% CI 10.9-13.7) were similar in patients from China to the overall population. Among patients from China who had dose reductions, TTSP was numerically longer than in those who did not (16.4 vs. 13.8 months; $P=0.0703$ ), while PFS was significantly longer (13.9 vs. 11.1 months; $P=0.0275$ ). Among patients from China with brain metastases, TTSP was numerically shorter than in those without (11.0 vs. 14.4 months; $P=0.0869)$, whereas PFS was significantly shorter (9.2 vs. 12.9 months; $P=0.0075$ ).

Conclusions Safety data for afatinib when used in a "near real-world" setting in patients with EGFRm+ NSCLC was consistent with the known safety profile of afatinib. Supporting efficacy data of afatinib were provided in all patients, and in those enrolled in China. Tolerability-guided afatinib dose reduction allowed patients to remain on treatment and continue to experience clinical benefit.
\end{abstract}

Trial Registration Number and Date of Registration NCT01953913 (1 October 2013).

Hai-Yan Tu and Jifeng Feng contributed equally to the article.

Yi-Long Wu

syylwu@live.cn

Extended author information available on the last page of the article

\section{Introduction}

The development of targeted epidermal growth factor receptor (EGFR) tyrosine kinase inhibitors (TKIs) resulted in improved efficacy versus chemotherapy in patients with $E G F R$ mutation-positive $(E G F R \mathrm{~m}+)$ non-small-cell lung cancer (NSCLC) [1-7], and these agents are recommended 


\section{Key Points}

In this prospective, phase IIIb, "near real-world" study, Asian patients with EGFR TKI-naïve EGFRm+ NSCLC were treated with afatinib.

Results were consistent with previously reported data for afatinib in this patient population, and no new safety signals were identified.

Additionally, patients who had afatinib dose reductions were able to stay on treatment.

first-line treatment options for patients with advanced EGFRm+ NSCLC [8-10].

EGFR mutations are particularly common among eastern Asian populations with NSCLC [11-14]. The secondgeneration TKI afatinib, an irreversible ErbB-family blocker, provides broader inhibition relative to first-generation TKIs, which may delay the development of acquired resistance [15]. In contrast to other EGFR TKIs, afatinib blocks signaling via all hetero- and homo-dimers formed by ErbB 1 (EGFR), but also ErbB2 (human epidermal growth factor receptor 2 (HER2)), ErbB3 (HER3), and ErbB4 (HER4) $[16,17]$.

In the phase III LUX-Lung 3 and LUX-Lung 6 trials in treatment-naïve patients with advanced EGFRm+ NSCLC, afatinib significantly improved progression-free survival (PFS) compared with platinum doublet therapy [6, 7]. In the phase IIb LUX-Lung 7 study, also conducted in treatmentnaïve patients with advanced $E G F R \mathrm{~m}+\mathrm{NSCLC}$, afatinib improved PFS as well as objective response rate (ORR) and time to treatment failure (TTF) compared with gefitinib [18]. Across these LUX-Lung trials, afatinib had a predictable and manageable safety profile, and few patients discontinued treatment because of adverse events (AEs) $[6,7,18]$. The clinical activity of afatinib has been shown in patients with brain metastases [19-21]; this is in contrast to dacomitinib, as patients with brain metastases were excluded from the phase III ARCHER 1050 trial [22].

As randomized controlled trials often have strict inclusion and exclusion criteria, performing trials that are more reflective of real-world clinical practice is crucial. Therefore, the aim of this study was to provide "near real-world" data on the safety and efficacy of afatinib in TKI-naïve Asian patients with locally advanced or metastatic EGFRm+ NSCLC. Interim results of the trial have been presented [23], and here we present final results for the overall population with a particular focus on data from the large subgroup of patients enrolled at study sites in China.

\section{Materials and Methods}

\subsection{Study Design and Patient Population}

This phase IIIb, multicenter, open-label, single-arm trial focused on the safety, tolerability, and efficacy of afatinib in a broad population of TKI-naïve Asian patients with locally advanced or metastatic EGFRm+ NSCLC (NCT01953913).

The primary safety endpoint was the number of patients with serious AEs (SAEs) and the secondary safety endpoint was the number of patients with AEs assessed by the treating physician as related to afatinib [i.e., treatment-related AEs (TRAEs)]. Various other safety endpoints were also evaluated. Although there were no primary or key secondary efficacy endpoints in the trial, time to symptomatic progression (TTSP) and PFS were selected to reflect real-world clinical practice and current treatment guidelines. These parameters were evaluated in the overall population and in the subgroup of patients in China. Tumor response (i.e., ORR) was also evaluated.

The study was conducted at 34 centers in China, Hong Kong, India, Singapore, and Taiwan. Inclusion and exclusion criteria were relatively broad to reflect a real-world setting. Inclusion criteria included age $\geq 18$ years (up to age 75 years in India only), with locally advanced or metastatic EGFRm+ NSCLC, and Eastern Cooperative Oncology Group performance status (ECOG PS) score of 0-2. Exclusion criteria included: previous use of EGFR TKI therapy; use of any anti-cancer medication $<2$ weeks, radiotherapy $<4$ weeks (except for palliative treatment), and major surgery $<4$ weeks prior to starting afatinib; history or presence of cardiovascular abnormalities; and symptomatic brain metastases.

The initial dosage regimen of afatinib was $40 \mathrm{mg}$ orally once daily, which was continued until tumor progression, lack of clinical benefit, or poor tolerability. Afatinib could be continued beyond radiologic progression for as long as the clinical investigator deemed that the patient was benefiting from treatment.

Treatment interruptions and dose reductions with afatinib could be implemented when necessary for the management of TRAEs, as follows: any drug-related grade $\geq 3 \mathrm{AE}$; grade $\geq 2$ diarrhea persisting for $\geq 48 \mathrm{~h}$ despite adequate treatment; reduced renal function (grade $\geq 2$ ), as measured by serum creatinine, proteinuria or $>50 \%$ reduction in glomerular filtration rate versus baseline. In cases where treatment interruption was required, afatinib was suspended until AE severity recovered to grade $\leq 1$, or to baseline severity. If recovery was achieved within 6 weeks, afatinib was resumed at a lower dose (decreased by $10 \mathrm{mg} /$ day decrements to a minimum of $20 \mathrm{mg} /$ day); if the patients could not tolerate $20 \mathrm{mg} /$ day, permanent discontinuation of afatinib was to be considered. Afatinib dose could not be increased after a dose reduction. 


\subsection{Assessments and Statistical Analyses}

The incidences and intensities of AEs, including SAEs, were monitored on a regular basis, including a daily record of diarrhea status. AE severity was classified according to the Common Terminology Criteria for Adverse Events v3.0, and the relationship of AEs to the study drug was determined by the investigators. TTSP was defined as the time from the date of the first dose of afatinib to the date of first documented clinically significant symptomatic progression necessitating a change in or discontinuation of cancer therapy. PFS was defined as the time from the date of the first dose of afatinib to the date of first documented radiologic and/or symptomatic progression according to the investigator's assessment or death, whichever came first. Both TTSP and PFS were assessed by the investigators. Radiologic assessments of tumors were conducted at the discretion of investigators, as per local standards. Patients with missing data were censored at the date of first administration.

Kaplan-Meier estimates and 95\% confidence intervals (CIs) for the median of the survival distribution were calculated for TTSP and PFS using Greenwood's standard error estimate. A post hoc analysis of efficacy outcomes (TTSP and PFS) was conducted for various subgroups including by age, ECOG PS, common/uncommon EGFR mutations, presence/absence of brain metastasis at baseline, and afatinib dose adjustment during the first 6 months of treatment.

\section{Results}

\subsection{Patients, Treatment Exposure, and Dose Reductions}

\subsubsection{Overall Population}

Of the 577 patients enrolled in the trial, 541 entered the study and were treated with afatinib (Fig. 1). Thus, demographic data for the overall population are based on these 541 patients who received the study drug. Recruitment of patients was between October 2013 and May 2017 and stopped once the recruitment goal was met. Baseline characteristics were generally representative of patients with EGFRm+ advanced NSCLC (Table 1).

The median duration of afatinib treatment was 12.9 months (386.0 days), with a range from 0.2 to 53.2 months (5 to 1596 days). Most patients $(\mathrm{n}=300 ; 55.5 \%)$ received afatinib as first-line therapy. Afatinib dose reductions from $40 \mathrm{mg} /$ day to $30 \mathrm{mg} /$ day were performed in 155 patients $(28.7 \%)$, and $36(6.7 \%)$ of these patients had their dose reduced further to $20 \mathrm{mg} /$ day. The median duration of

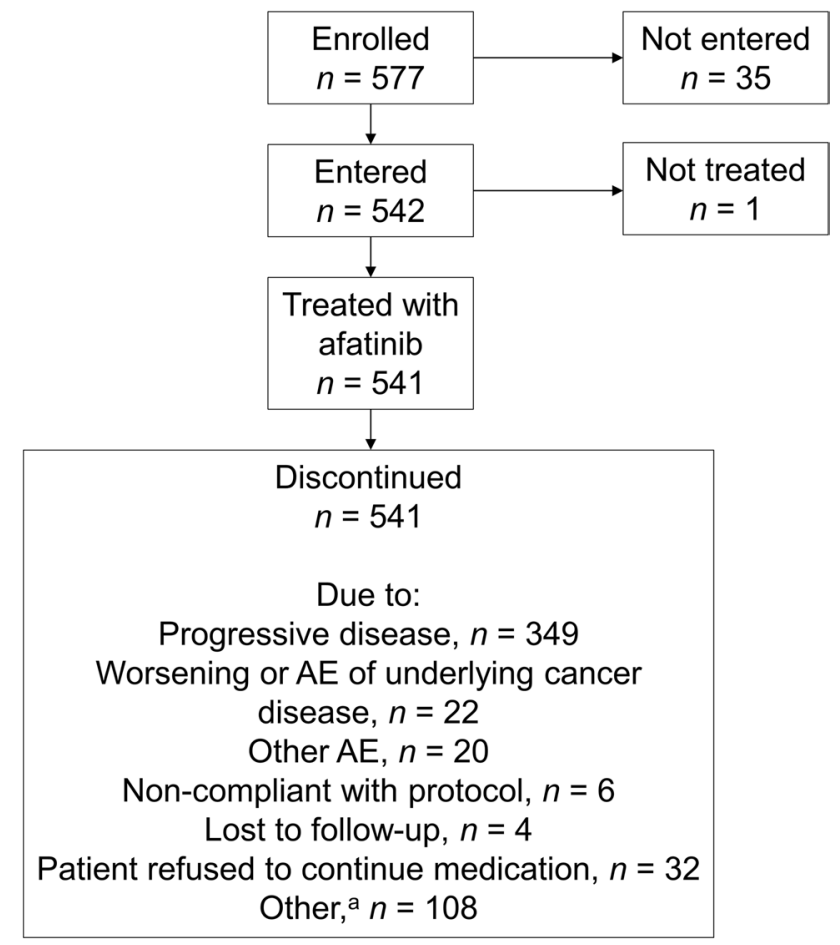

Fig. 1 Disposition diagram. ancludes patients who switched to commercial drug. $A E$ adverse event

treatment with afatinib 40, 30, and $20 \mathrm{mg} /$ day was 253.0, 265.0 , and 225.5 days, respectively.

\subsubsection{Subgroup of Patients Enrolled in China}

From the overall population of 541 patients, 412 (76.2\%) were enrolled at 12 sites in China (Table 1). In general, patient demographics and afatinib treatment patterns were similar between the patients enrolled in China and the overall population. Median age was 59.0 years (range 32-81), $54.4 \%$ were female, and $67.7 \%$ of patients had never smoked. Common EGFR mutations (Del19 and L858R), with or without uncommon EGFR mutations, were harbored by $87.4 \%$ of patients; uncommon $E G F R$ mutations only were reported in $12.6 \%$ of patients. ECOG PS score was 0 or 1 in $99 \%$ of patients, and $20.4 \%$ of patients had brain metastases.

The median duration of afatinib treatment in patients enrolled in China with afatinib 40, 30, and $20 \mathrm{mg}$ /day was 277, 266, and 229 days, respectively. Afatinib dose reductions from $40 \mathrm{mg} /$ day to $30 \mathrm{mg} /$ day occurred in 73 patients (17.7\%), and $19(4.6 \%)$ of these patients had their dose reduced further to $20 \mathrm{mg} / \mathrm{day}$. The median time to first dose reduction was 56 days (range 12-1273). 
Table 1 Baseline demographic and clinical characteristics of the overall population and subgroup of patients enrolled at sites in China

\begin{tabular}{|c|c|c|}
\hline Parameter & Overall population $(N=541)$ & $\begin{array}{l}\text { Subgroup enrolled at sites } \\
\text { in China }(n=412)\end{array}$ \\
\hline \multicolumn{3}{|l|}{ Age, $n(\%)$} \\
\hline Years, median (range) & $59.0(27-82)$ & $59.0(32-81)$ \\
\hline$\geq 65$ years, $n(\%)$ & $147(27.2)$ & $105(25.5)$ \\
\hline$<65$ years, $n(\%)$ & $394(72.8)$ & $307(74.5)$ \\
\hline Female, $n(\%)$ & $286(52.9)$ & $224(54.4)$ \\
\hline \multicolumn{3}{|l|}{ Country, $n(\%)$} \\
\hline China & $412(76.2)$ & $412(100)$ \\
\hline Hong Kong & $25(4.6)$ & \\
\hline India & $50(9.2)$ & \\
\hline Singapore & $24(4.4)$ & \\
\hline Taiwan & $30(5.5)$ & \\
\hline \multicolumn{3}{|l|}{ Smoking status, $n(\%)$} \\
\hline Current smoker & $29(5.4)$ & $23(5.6)$ \\
\hline Ex-smoker & $137(25.3)$ & $110(26.7)$ \\
\hline Never smoked & $375(69.3)$ & $279(67.7)$ \\
\hline \multicolumn{3}{|l|}{ Tumor histology, $n(\%)$} \\
\hline Adenocarcinoma & $517(95.6)$ & $399(96.8)$ \\
\hline Squamous cell carcinoma & $12(2.2)$ & $11(2.7)$ \\
\hline Other & $12(2.2)$ & $2(0.5)$ \\
\hline \multicolumn{3}{|l|}{ EGFR mutation, $n(\%)^{\mathrm{a}}$} \\
\hline Common, with or without uncommon ${ }^{\text {b }}$ & $477(88.2)$ & $360(87.4)$ \\
\hline Uncommon only ${ }^{\mathrm{c}}$ & $64(11.8)$ & $52(12.6)$ \\
\hline Del19 & $261(48.2)$ & $178(43.2)$ \\
\hline L858R & $219(40.5)$ & $184(44.7)$ \\
\hline Exon 20 insertion & $30(5.6)$ & $26(6.3)$ \\
\hline L861Q & $31(5.7)$ & $29(7.0)$ \\
\hline G719X & $25(4.6)$ & $23(5.6)$ \\
\hline S768I & $11(2.0)$ & $10(2.4)$ \\
\hline $\mathrm{T} 790 \mathrm{M}$ & $6(1.1)$ & $6(1.5)$ \\
\hline Other & $7(1.3)$ & $2(0.5)$ \\
\hline \multicolumn{3}{|l|}{ ECOG PS score, $n(\%)$} \\
\hline 0 & $99(18.3)$ & $62(15.0)$ \\
\hline 1 & $431(79.7)$ & $346(84.0)$ \\
\hline 2 & $11(2.0)$ & $4(1.0)$ \\
\hline Brain metastases, $n(\%)$ & $103(19.0)$ & $84(20.4)$ \\
\hline \multicolumn{3}{|l|}{ Therapy line of afatinib, $n(\%)$} \\
\hline First & $300(55.5)$ & $243(59.0)$ \\
\hline Second & $158(29.2)$ & $123(29.9)$ \\
\hline Third or later & $53(9.8)$ & $46(11.2)$ \\
\hline Prior anti-cancer therapies, $n(\%)^{\mathrm{d}}$ & $281(51.9)$ & $213(51.7)$ \\
\hline Surgery & $122(22.6)$ & $100(24.3)$ \\
\hline Radiotherapy & $64(11.8)$ & $39(9.5)$ \\
\hline Other & $215(39.7)$ & $172(41.7)$ \\
\hline Chemotherapy & $211(39.0)$ & $171(41.5)$ \\
\hline
\end{tabular}

ECOG PS Eastern Cooperative Oncology Group performance status, EGFR epidermal growth factor receptor

a Patients can appear in more than one mutation category

${ }^{\mathrm{b}}$ Patients with EGFR mutation categories: Del19 and/or L858R, and with or without uncommon EGFR mutation

${ }^{c}$ Patients with mutation categories other than Del19 and/or L858R

${ }^{\mathrm{d}}$ Patients may have more than one prior therapy recorded 


\subsection{Safety and Tolerability}

\subsubsection{Overall Population}

Almost all patients $(98.7 \%)$ treated with afatinib experienced an AE, although most (54.2\%) were grade 1 or 2 . Similarly, almost all patients $(97.6 \%)$ had a TRAE. The three most common AEs were diarrhea (90.2\% of patients), rash/acne (77.1\%), and stomatitis (58.8\%). These were also the three most common any grade/grade $\geq 3$ TRAEs (diarrhea: $89.6 \% / 10.9 \%$; rash/acne: $76.5 \% / 8.7 \%$; stomatitis: 58.4\%/4.3\%; Fig. 2a).

A total of 154 patients $(28.5 \%)$ experienced an $\mathrm{AE}$ leading to a reduction in afatinib dose. The most frequent AEs resulting in a dose reduction were diarrhea $(11.1 \%$ of patients) and rash/acne (9.6\%). Overall, 47 patients (8.7\%) experienced AEs leading to treatment discontinuation, although TRAEs leading to treatment discontinuation were reported in only 17 patients (3.1\%).
Overall, 164 patients $(30.3 \%)$ had SAEs, including $58(10.7 \%), 24(4.4 \%)$, and $46(8.5 \%)$ patients with SAEs of grade 3,4 , and 5 (fatal), respectively. The most frequently reported SAEs included pleural effusion, dyspnea, and diarrhea (Fig. 2b). Although 46 patients had AEs with fatal outcomes, a number of the deaths could be attributed to disease progression, and only three cases $(0.6 \%)$ were assessed by investigators as drug related; these deaths were attributed to decreased appetite, dyspnea, and respiratory failure.

\subsubsection{Subgroup of Patients Enrolled in China}

In general, safety and tolerability data for the patients enrolled in China were similar to data reported for the overall population. The proportion of patients enrolled in China treated with afatinib who experienced any $\mathrm{AE}$ was $99.3 \%$, although most were grade 1 or $2(61.7 \%)$. As was the case in the overall population, almost all patients enrolled in China $(97.8 \%)$ had a TRAE. Similarly, the most frequent TRAEs
Fig. 2 a Most frequently reported treatment-related adverse events (TRAEs) of any grade and grade $\geq 3$ intensity; b SAEs reported in the overall population. ${ }^{\mathrm{a}}$ Grouped terms. $A L T$ alanine aminotransferase, AST aspartate aminotransferase, $C N S$ central nervous system, $P P E$ palmar-plantar erythrodysesthesia, SAEs serious adverse events

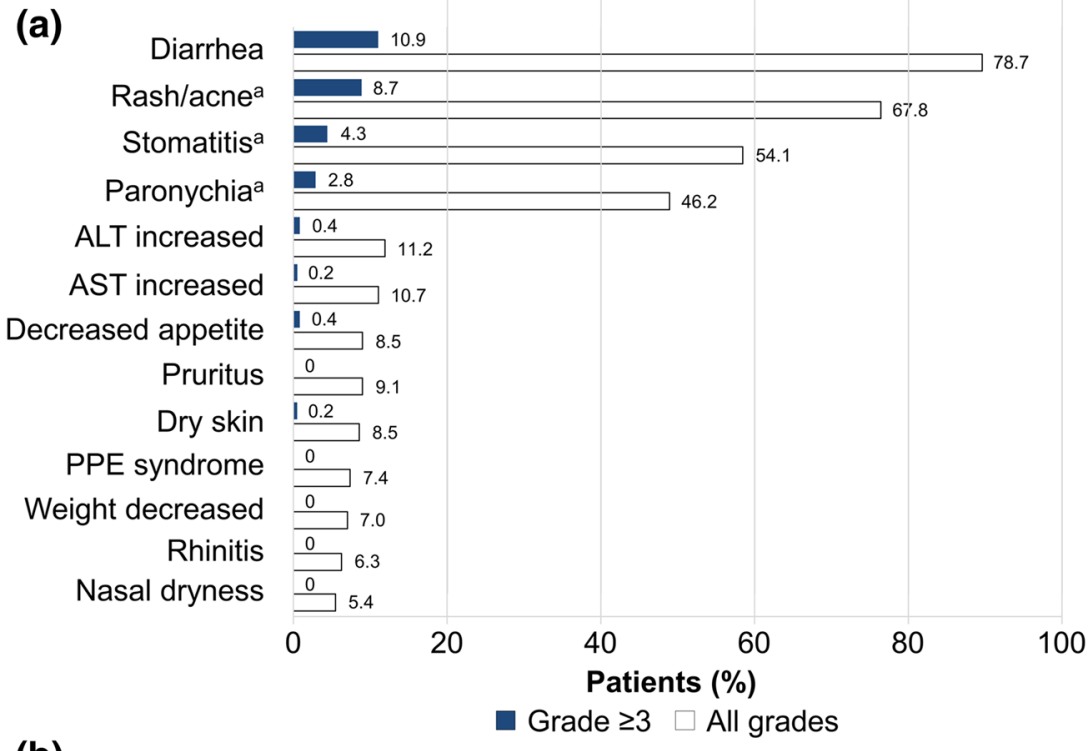

(b)

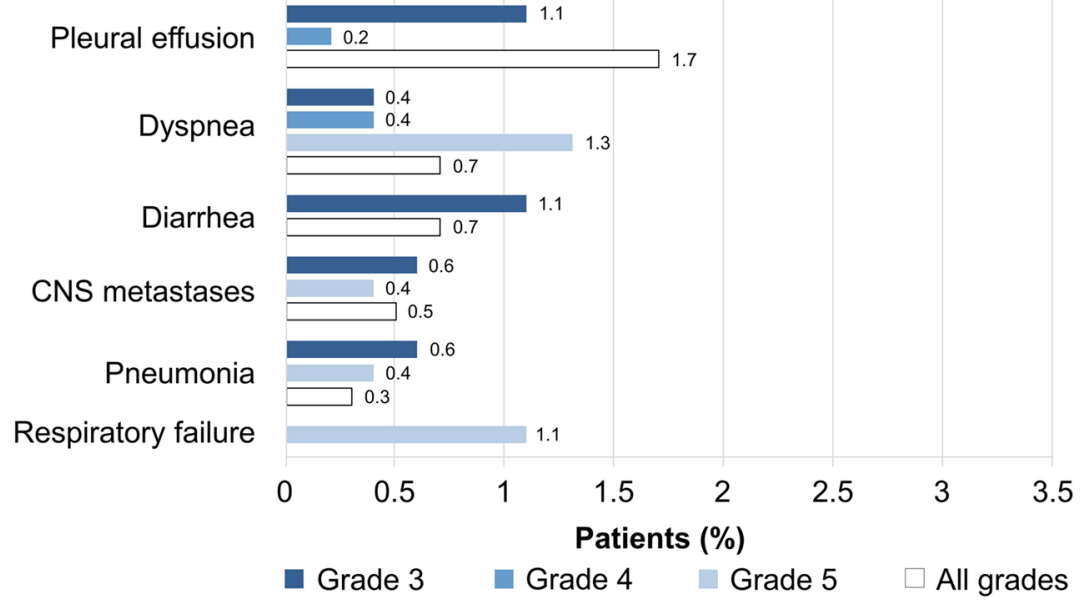


Table 2 Most frequently reported treatment-related adverse events (TRAEs) ${ }^{\mathrm{a}}$ in patients enrolled in China before and after tolerabilityguided dose reductions

\begin{tabular}{|c|c|c|c|c|}
\hline \multirow[t]{2}{*}{ TRAE } & \multicolumn{2}{|c|}{ Before dose reduction } & \multicolumn{2}{|c|}{ After dose reduction } \\
\hline & $\begin{array}{l}\text { Grade } \\
\geq 3\end{array}$ & Any grade & $\begin{array}{l}\text { Grade } \\
\geq 3\end{array}$ & Any grade \\
\hline Diarrhea, $\%$ & 26.0 & 95.5 & 3.9 & 51.3 \\
\hline Rash/acne, $\%^{\mathrm{b}}$ & 23.4 & 70.1 & 10.4 & 58.4 \\
\hline Stomatitis, $\%^{\mathrm{b}}$ & 13.0 & 64.3 & 4.5 & 43.5 \\
\hline Paronychia, $\%^{\mathrm{b}}$ & 7.8 & 39.0 & 3.3 & 43.5 \\
\hline Decreased appetite, $\%$ & 0.6 & 11.7 & 1.3 & 11.0 \\
\hline
\end{tabular}

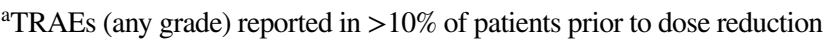
${ }^{\mathrm{b}}$ Grouped term

grade $\geq 3$ were diarrhea $(8.0 \%)$, rash/acne $(6.8 \%)$, stomatitis $(3.2 \%)$, and paronychia (1.9\%).

SAEs were reported in 104 patients $(25.2 \%)$ enrolled in China, including 34 (8.3\%), 19 (4.6\%), and 28 (6.8\%) with SAEs of grade 3, 4, and 5, respectively. The most frequently reported SAEs included dyspnea, pleural effusion, and respiratory failure. Among this subgroup, 92 patients (22.3\%) experienced an AE leading to dose reduction, most commonly diarrhea $(9.2 \%)$ and rash $(7.5 \%)$. Any grade TRAEs, and grade $\geq 3$ TRAEs in particular, were dramatically reduced by afatinib dose reduction (Table 2 ). Only

(a)

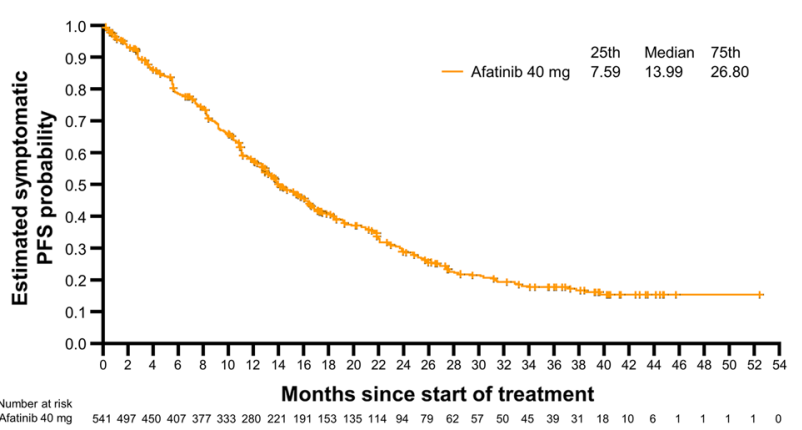

(c)

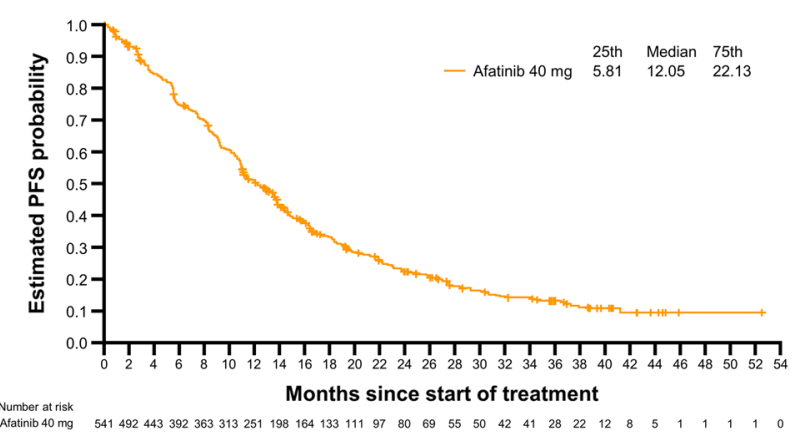

11 patients $(2.7 \%)$ had a drug-related AE leading to treatment discontinuation. Paronychia, anemia, and rash were the reasons for treatment discontinuation in two patients each $(0.5 \%)$; other AEs leading to discontinuation were reported in only one patient each. A total of 19 patients $(4.6 \%)$ were deemed to have a drug-related SAE; ten, three, and three patients had grade 3, 4, and 5 drug-related SAEs, respectively. Of the patients enrolled in China, 28 had AEs leading to death, although only three cases were assessed by investigators as drug related; these deaths were attributed to decreased appetite, dyspnea, and respiratory failure.

\subsection{Time to Symptomatic Progression (TTSP), Progression-Free Survival (PFS), Objective Response Rate (ORR), and Post Hoc Subgroup Analyses}

\subsubsection{Overall Population}

The overall population of 541 patients included those with previous chemotherapy treatment, uncommon mutations, and brain metastases. A total of 371 patients $(68.6 \%)$ had clinically meaningful symptomatic progression during the study period, and the median TTSP was 14.0 months (95\% CI 12.9-15.9; Fig. 3). A total of 416 patients (76.9\%) progressed or died during the study, and the median PFS was 12.1 months (95\% CI 11.0-13.6; Fig. 3). (b)

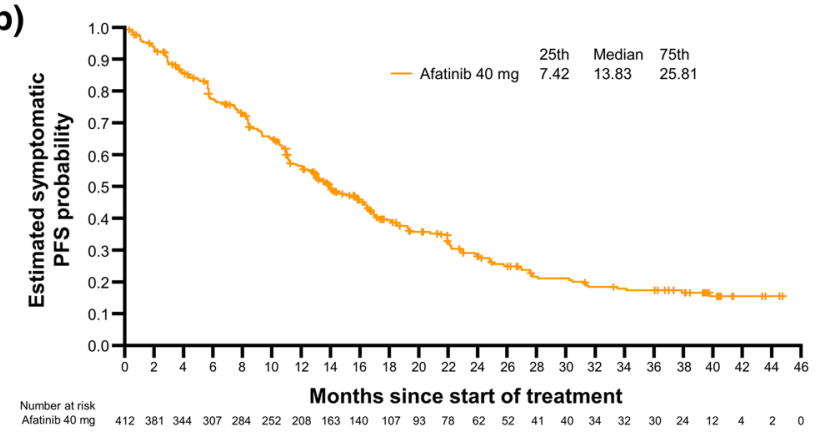

(d)

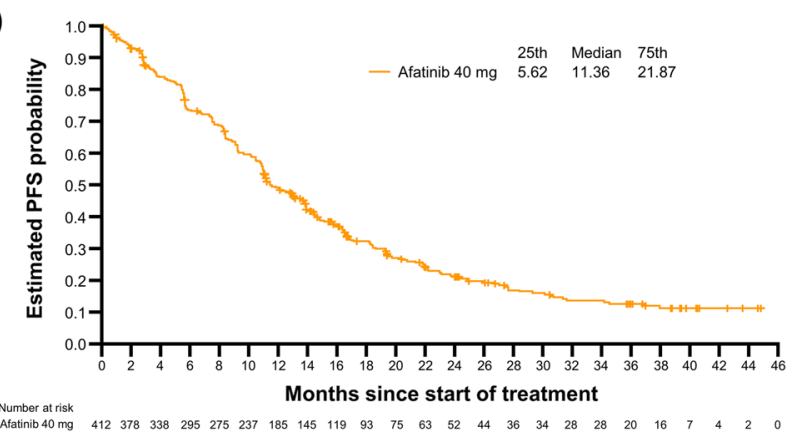

Fig. 3 Kaplan-Meier curves for a TTSP in the overall population; b TTSP in patients enrolled in China; $\mathbf{c}$ PFS in the overall population; and d PFS in patients enrolled in China. PFS progression-free survival, TTSP time to symptomatic progression 
The ORR for the overall population was $59.1 \%$ (95\% CI 54.9-63.3). A total of 13 patients (2.4\%) achieved a complete response and 307 patients (56.7\%) had a partial response. The median duration of objective response was 12.2 months (95\% CI 11.1-13.5). Results of the post hoc subgroup analyses for TTSP and PFS are presented in Table 3. Both TTSP and PFS were numerically longer in patients with common versus uncommon EGFR mutations, in elderly ( $\geq 65$ years of age) versus younger $(<65$ years of age) patients, in patients with an ECOG PS of 0 versus 1, in patients without versus those with brain metastases at baseline, and in patients with afatinib dose reductions from $40 \mathrm{mg}$ in the first 6 months versus those without dose reductions.

\subsubsection{Subgroup of Patients Enrolled in China}

Results of the post hoc subgroup analyses for TTSP and PFS in patients enrolled in China are presented in Table 3. The median TTSP was 13.8 months (95\% CI 12.7-16.1; Fig. 3), which was nearly the same as the median TTSP of 14.0 months reported in the overall population. Similar to the overall population, median TTSP in patients enrolled in China was numerically longer in patients with common versus uncommon $E G F R$ mutations (14.3 vs. 9.2 months) and among elderly versus patients aged $<65$ years old (16.1 vs. 13.5 months). Median TTSP was similar among patients with ECOG PS of 0 or 1 (14.3 vs. 13.8 months). PFS results in patients enrolled in China were comparable to those in the overall population; 310 patients $(75.2 \%)$ progressed or died during the study, and the median PFS was 11.4 months (95\% CI 10.9-13.7).

A total of 92 of the patients enrolled in China had their afatinib dose reduced (22.3\%), 66 of whom had reductions during the first 6 months of treatment. TTSP in patients who had a dose reduction in the first 6 months of treatment was 16.4 months compared with 13.8 months in patients who did not have a dose reduction (hazard ratio (HR) 1.38; 95\% CI 0.97-1.96; $P=0.0703$ ) (Electronic Supplementary Material (ESM), Fig. S1). PFS in patients who had a dose reduction was significantly longer at 13.9 months compared with 11.1 months in patients who did not have a dose reduction (HR 1.46; 95\% CI 1.04-2.04; $P=0.0275$ ) (ESM, Fig. S1).

Among the 84 patients enrolled in China with brain metastases at baseline, TTSP was 11.0 months compared with 14.4 months in those without brain metastases (HR 1.29; 95\% CI 0.96-1.73; $P=0.0869$; ESM, Fig. S1). PFS was significantly shorter in patients with brain metastases compared with those without (9.2 vs. 12.9 months; HR $1.45 ; 95 \%$ CI 1.10-1.90; $P=0.0075$; ESM, Fig. S1).

\section{Discussion}

Results of this prospective "near real-world" study conducted in several Asian countries were consistent with previously reported data for afatinib in TKI-naïve patients with locally advanced or metastatic EGFRm+ NSCLC and did not identify any new safety signals. Evaluating drugs in real-world settings is important because naturalistic studies can generate data relevant to a broad range of patients, as is typically seen in clinical practice.

Although the baseline demographics and clinical characteristics of this study group were more diverse to emulate a real-world setting, safety data were generally consistent with those from the randomized LUX-Lung 3, 6, and 7 trials with afatinib; the most frequently reported TRAEs (diarrhea, rash/acne, stomatitis) are recognized AEs of afatinib and EGFR TKIs [6, 7, 18].

Although the overall rate of afatinib dose reduction in the current study was similar to that in the LUX-Lung 6 study [7], it was lower than that reported in LUX-Lung 3 and $7[6,18]$, which may reflect differences in study populations and/or management of AEs such as diarrhea. There was a low rate of discontinuation due to TRAEs in the current study (as well as in the LUX-Lung 3, 6, and 7 trials), which suggests that TRAEs with afatinib may be managed by dose reduction while maintaining therapeutic efficacy. This was also observed in the subgroup of patients enrolled in China, in which the number and severity of TRAEs after dose reduction was dramatically reduced. In addition, TTSP was longer, but not significantly, and PFS was significantly longer in patients who had their dose reduced compared with those who did not. This supports previous findings from the LUX-Lung 3 and 6 trials, which found no negative effect of dose reduction on PFS [18], and shows that timely dose reduction can allow patients to remain on therapy for longer, while continuing to derive clinical benefit.

Findings of a global, non-interventional, real-world study (RealGiDo) with afatinib provide support to these findings, in that the use of dose reductions reduced the intensity and frequency of AEs, but did not compromise clinical effectiveness [24]. Other studies have evaluated afatinib in routine clinical practice, showing similar tolerability profiles and safety findings to those observed in the current study [25-27].

Median PFS in the overall population in the current study was approximately 12 months and median TTSP was approximately 14 months. These findings suggest that afatinib treatment may be continued beyond disease progression. Similarly, in LUX-Lung 7, in which $35 \%$ of patients received afatinib beyond radiologic progression, median TTF was almost 3 months longer than median PFS (13.7 vs. 11.0 months) [18]. 
Table 3 Post hoc analysis of time to symptomatic progression (TTSP) and progression-free survival (PFS) for specified subgroups in overall population and Chinese centers

\begin{tabular}{|c|c|c|}
\hline & \multicolumn{2}{|l|}{ Overall population } \\
\hline & \multicolumn{2}{|l|}{ Subgroup } \\
\hline EGFR mutation type & Common & Uncommon only \\
\hline$N$ & 477 & 64 \\
\hline Median TTSP, months (95\% CI) & $14.42(13.40-16.39)$ & $9.23(6.01-20.66)$ \\
\hline Median PFS, months (95\% CI) & $12.68(11.10-13.83)$ & $9.06(5.58-12.94)$ \\
\hline Afatinib dose adjustment & $40 \mathrm{mg}$ in first 6 months & $<40 \mathrm{mg}$ in first 6 months \\
\hline$N$ & 421 & 120 \\
\hline Median TTSP, months (95\% CI) & $13.83(12.51-15.67)$ & $16.12(12.94-23.02)$ \\
\hline Median PFS, months (95\% CI) & $11.33(10.90-13.53)$ & $13.86(10.90-16.52)$ \\
\hline Baseline brain metastasis & With & Without \\
\hline$N$ & 103 & 438 \\
\hline Median TTSP, months (95\% CI) & $12.78(10.08-15.63)$ & $14.42(13.14-16.52)$ \\
\hline Median PFS, months (95\% CI) & $10.08(8.31-12.05)$ & $12.94(11.30-14.09)$ \\
\hline Age & $<65$ years & $\geq 65$ years \\
\hline$N$ & 394 & 147 \\
\hline Median TTSP, months (95\% CI) & $13.63(12.22-15.27)$ & $17.01(13.69-21.71)$ \\
\hline Median PFS, months (95\% CI) & $11.53(10.87-13.56)$ & $13.56(11.07-15.67)$ \\
\hline ECOG PS & ECOG 0 & ECOG 1 \\
\hline$N$ & 99 & 431 \\
\hline Median TTSP, months (95\% CI) & $16.42(13.04-21.87)$ & $13.76(12.22-15.63)$ \\
\hline \multirow[t]{3}{*}{ Median PFS, months (95\% CI) } & $14.84(12.25-19.31)$ & $11.33(10.87-13.04)$ \\
\hline & \multicolumn{2}{|l|}{ Chinese centers } \\
\hline & \multicolumn{2}{|l|}{ Subgroup } \\
\hline EGFR mutation type & Common & Uncommon only \\
\hline$N$ & 360 & 52 \\
\hline Median TTSP, months (95\% CI) & $14.32(12.91-16.42)$ & $9.23(5.68-20.66)$ \\
\hline Median PFS, months (95\% CI) & $12.02(10.94-13.83)$ & $9.06(5.58-16.58)$ \\
\hline Afatinib dose adjustment & $40 \mathrm{mg}$ in first 6 months & $<40 \mathrm{mg}$ in first 6 months \\
\hline$N$ & 346 & 66 \\
\hline Median TTSP, months (95\% CI) & $13.76(11.30-16.09)$ & $16.39(12.91-27.59)$ \\
\hline Median PFS, months (95\% CI) & $11.10(10.77-12.97)$ & $13.86(10.94-19.31)$ \\
\hline Baseline brain metastasis & With & Without \\
\hline$N$ & 84 & 328 \\
\hline Median TTSP, months (95\% CI) & $11.03(9.03-15.63)$ & $14.35(12.91-16.58)$ \\
\hline Median PFS, months (95\% CI) & $9.20(7.45-11.07)$ & $12.94(11.10-14.42)$ \\
\hline Age & $<65$ years & $\geq 65$ years \\
\hline$N$ & 307 & 105 \\
\hline Median TTSP, months (95\% CI) & $13.53(11.53-15.93)$ & $16.09(12.68-22.00)$ \\
\hline Median PFS, months (95\% CI) & $11.33(10.64-13.56)$ & $12.97(10.90-15.70)$ \\
\hline ECOG PS & ECOG 0 & ECOG 1 \\
\hline$N$ & 62 & 346 \\
\hline Median TTSP, months (95\% CI) & $14.32(8.31-19.21)$ & $13.79(12.02-15.93)$ \\
\hline Median PFS, months (95\% CI) & $13.56(8.31-19.21)$ & $11.33(10.90-13.56)$ \\
\hline
\end{tabular}

CI confidence interval, ECOG PS Eastern Cooperative Oncology Group performance status, EGFR epidermal growth factor receptor 
Around three-quarters of the overall population were enrolled in centers in China, and subgroup analyses showed similar results for these patients compared with the overall population. The median PFS here (11.4 months) was similar to PFS values observed in two other studies enrolling Chinese patients (12.3 and 14.2 months, respectively) [28, 29]. Studies recruiting patients from Taiwan also had similar PFS values (11.8, 12.4, and 12.2 months, respectively) [30-32]. However, patients in this study had lower median PFS than that reported in a retrospective real-world study of patients in Korea (15.1 months) [33].

TTSP and PFS were shorter, the latter significantly so, in patients enrolled in China with baseline brain metastases compared with those without. This may be expected, as these patients are likely to be more unwell than patients without brain metastases, and has been observed previously in the LUX-Lung 3 and 6 studies [19]. However, it should be noted that patients with brain metastases in this analysis and in the LUX-Lung studies still gained substantial clinical benefits from afatinib treatment, and in the LUX-Lung studies this benefit was greater than that observed with platinumbased chemotherapy [19].

The use of osimertinib as a first-line treatment option in patients with $E G F R \mathrm{~m}+$ (Del19 or L858R) NSCLC is now established, based on results showing improved efficacy versus first-generation EGFR TKIs in the Phase III FLAURA trial [34]. Notably, however, OS results were not shown to be statistically significant in Asian patients receiving osimertinib versus those receiving first-generation EGFR TKIs [35]. Afatinib also remains an important treatment choice in patients with uncommon EGFR mutations (accounting for around $10 \%$ of $E G F R$ mutations) [36], showing improved efficacy versus first-generation EGFR TKIs [37]. In a large database study of afatinib treatment in this patient group, median TTF was 10.8 months and ORR was $60.0 \%$ in those with "major" uncommon mutations (G719X, L861Q, and S768I) [38]. Interestingly, results from a Japanese retrospective cohort study showed a statistically significant survival advantage in patients with EGFR L858R mutations without brain metastases who were treated with afatinib versus osimertinib [39].

Most patients in this study received afatinib as first-line therapy $(55.5 \%)$. First-line treatment with afatinib expands the number of available options for patients, often meaning that chemotherapy can be postponed until later lines. Results from the global real-world GioTag study demonstrated that sequential treatment with afatinib followed by osimertinib was associated with encouraging outcomes in patients with $E G F R \mathrm{~m}+$ NSCLC, particularly in Del19-positive patients and Asian patients [40]. Resistance mechanisms to osimertinib are highly heterogenous [41], and thus there are currently no approved targeted treatment options following progression on the drug. Delaying osimertinib use to second-line treatment, after first-line afatinib treatment, may therefore maximize the length of time on targeted treatment.

The lack of a comparator treatment arm is a notable limitation of the current study. Furthermore, the subgroup analyses of TTSP and PFS, including in patients enrolled in China, were conducted post hoc, with the inherent disadvantages of this type of analysis. It may be seen as a limitation that over $75 \%$ of the patients were from one country, and, thus, the subgroup analysis of patients from China closely reflected the overall analysis. However, it is also useful to gain information on this large patient group from the world's most populous country.

\section{Conclusions}

Safety data for afatinib, when used in a "near real-world" setting in TKI-naïve Asian patients with locally advanced or metastatic NSCLC harboring EGFR mutations, were consistent with previously reported data and did not identify any new safety signals in either the overall population or the large subgroup of patients from China. For most patients, AEs were manageable and did not require discontinuation of afatinib. The study also provides supporting efficacy data with afatinib in this setting, including "near real-world" data on efficacy in a large population of Chinese patients.

Supplementary Information The online version contains supplementary material available at https://doi.org/10.1007/s11523-021-00859-6.

Acknowledgements This study was funded by Boehringer Ingelheim Inc. Medical writing support for the development of this manuscript, under the direction of the authors, was provided by Steven Kirkham, $\mathrm{PhD}$, of Ashfield MedComms, an Ashfield Health company, and was funded by Boehringer Ingelheim Inc. The authors received no direct compensation related to the development of the manuscript.

\section{Declarations}

Funding This work was supported by Boehringer Ingelheim International $\mathrm{GmbH}$. The study sponsor participated in the design of the study, the collection, analysis, and interpretation of the data, writing the article, and the decision to submit the article for publication.

Conflicts of interest Y-LW reports receiving honoraria from AstraZeneca, Boehringer Ingelheim, Bristol Myers Squibb, Eli Lilly, Hengrui, Merck Sharp \& Dohme, Pfizer, Roche, Sanofi; and receiving grants or funds from AstraZeneca, BMS, and Pfizer. KCL declares receiving honoraria (outside the submitted work) from Pifzer, AstraZeneca, Eli Lily, Roche. YT is employed by Boehringer Ingelheim (China) Investment Co., Ltd. ZX is employed by Boehringer Ingelheim (China) Investment Co., Ltd. AC was employed by Boehringer Ingelheim International GmbH. DC-LH was employed by Boehringer Ingelheim Taiwan Limited and is currently employed with Merck Sharp \& Dohme (I.A.) LLC, Taiwan Branch. All the remaining authors declare no potential conflicts of interest. 
Ethics approval At each participating center, the study was approved by the Institutional Review Board or Independent Ethics Committee, and was conducted in accordance with the Declaration of Helsinki, the International Conference on Harmonisation of Technical Requirements for Pharmaceuticals for Human Use, Good Clinical Practice, and local laws. All patients provided written informed consent.

Consent for publication All patients provided written informed consent.

Availability of data and material To ensure independent interpretation of clinical study results, Boehringer Ingelheim grants all external authors access to relevant material, including participant-level clinical study data, as needed by them to fulfill their role and obligations as authors under the ICMJE criteria. Clinical study documents and participant clinical study data are available to be shared on request after publication of the primary manuscript in a peer-reviewed journal, and if regulatory activities are complete and other criteria met as per the BI Policy on Transparency and Publication of Clinical Study Data (see https://www.mystudywindow.com/msw/datasharing). Bona fide, qualified scientific and medical researchers are eligible to request access to the clinical study data with corresponding documentation describing the structure and content of the datasets. Upon approval, and governed by a Legal Agreement, data are shared in a secured data-access system for a limited period of 1 year, which may be extended upon request. Prior to providing access, clinical study documents and data will be examined, and, if necessary, redacted and de-identified, to protect the personal data of study participants and personnel, and to respect the boundaries of the informed consent of the study participants. Researchers should use the https://vivli.org/ link to request access to study data and visit https://www.mystudywindow.com/msw/datasharing for further information.

Author contributions All authors critically reviewed and drafted the manuscript, and agreed to be accountable for all aspects of the work. AC contributed to the development of the draft at all earlier stages, all other authors provided feedback on the final version of the manuscript.

Open Access This article is licensed under a Creative Commons Attribution-NonCommercial 4.0 International License, which permits any non-commercial use, sharing, adaptation, distribution and reproduction in any medium or format, as long as you give appropriate credit to the original author(s) and the source, provide a link to the Creative Commons licence, and indicate if changes were made. The images or other third party material in this article are included in the article's Creative Commons licence, unless indicated otherwise in a credit line to the material. If material is not included in the article's Creative Commons licence and your intended use is not permitted by statutory regulation or exceeds the permitted use, you will need to obtain permission directly from the copyright holder. To view a copy of this licence, visit $\mathrm{http} / / /$ creativecommons.org/licenses/by-nc/4.0/.

\section{References}

1. Mok TS, Wu YL, Thongprasert S, Yang CH, Chu DT, Saijo N, et al. Gefitinib or carboplatin-paclitaxel in pulmonary adenocarcinoma. N Engl J Med. 2009;361(10):947-57. https://doi.org/10. 1056/NEJMoa0810699.

2. Rosell R, Carcereny E, Gervais R, Vergnenegre A, Massuti B, Felip E, et al. Erlotinib versus standard chemotherapy as firstline treatment for European patients with advanced EGFR mutation-positive non-small-cell lung cancer (EURTAC): a multicentre, open-label, randomised phase 3 trial. Lancet Oncol.
2012;13(3):239-46. https://doi.org/10.1016/S1470-2045(11) 70393-X.

3. Maemondo M, Inoue A, Kobayashi K, Sugawara S, Oizumi S, Isobe $\mathrm{H}$, et al. Gefitinib or chemotherapy for non-small-cell lung cancer with mutated EGFR. N Engl J Med. 2010;362(25):2380-8. https://doi.org/10.1056/NEJMoa0909530.

4. Zhou C, Wu YL, Chen G, Feng J, Liu XQ, Wang C, et al. Erlotinib versus chemotherapy as first-line treatment for patients with advanced EGFR mutation-positive non-small-cell lung cancer (OPTIMAL, CTONG-0802): a multicentre, open-label, randomised, phase 3 study. Lancet Oncol. 2011;12(8):735-42. https://doi.org/10.1016/S1470-2045(11)70184-X.

5. Wu YL, Zhou C, Liam CK, Wu G, Liu X, Zhong Z, et al. First-line erlotinib versus gemcitabine/cisplatin in patients with advanced EGFR mutation-positive non-small-cell lung cancer: analyses from the phase III, randomized, open-label, ENSURE study. Ann Oncol. 2015;26(9):1883-9. https://doi.org/10.1093/annonc/ $\operatorname{mdv} 270$.

6. Sequist LV, Yang JC, Yamamoto N, O’Byrne K, Hirsh V, Mok T, et al. Phase III study of afatinib or cisplatin plus pemetrexed in patients with metastatic lung adenocarcinoma with EGFR mutations. J Clin Oncol. 2013;31(27):3327-34. https://doi.org/10. 1200/JCO.2012.44.2806.

7. Wu YL, Zhou C, Hu CP, Feng J, Lu S, Huang Y, et al. Afatinib versus cisplatin plus gemcitabine for first-line treatment of Asian patients with advanced non-small-cell lung cancer harbouring EGFR mutations (LUX-Lung 6): an open-label, randomised phase 3 trial. Lancet Oncol. 2014;15(2):213-22. https://doi.org/10.1016/ S1470-2045(13)70604-1.

8. Hanna N, Johnson D, Temin S, Baker S Jr, Brahmer J, Ellis PM, et al. Systemic therapy for stage IV non-small-cell lung cancer: American Society of Clinical Oncology clinical practice guideline update. J Clin Oncol. 2017;35(30):3484-515. https://doi.org/10. 1200/JCO.2017.74.6065.

9. Planchard D, Popat S, Kerr K, Novello S, Smit EF, Faivre-Finn $\mathrm{C}$, et al. Metastatic non-small cell lung cancer: ESMO Clinical Practice Guidelines for diagnosis, treatment and follow-up. Ann Oncol. 2018;29(Suppl4):iv192-237. https://doi.org/10.1093/ annonc/mdy 275 .

10. Wu YL, Planchard D, Lu S, Sun H, Yamamoto N, Kim DW, et al. Pan-Asian adapted Clinical Practice Guidelines for the management of patients with metastatic non-small-cell lung cancer: a CSCO-ESMO initiative endorsed by JSMO, KSMO, MOS, SSO and TOS. Ann Oncol. 2019;30(2):171-210. https://doi.org/10. 1093/annonc/mdy554.

11. Han B, Tjulandin S, Hagiwara K, Normanno N, Wulandari L, Laktionov K, et al. EGFR mutation prevalence in Asia-Pacific and Russian patients with advanced NSCLC of adenocarcinoma and non-adenocarcinoma histology: the IGNITE study. Lung Cancer. 2017;2017(113):37-44. https://doi.org/10.1016/j.lungcan.2017. 08.021 .

12. Shi Y, Au JS, Thongprasert S, Srinivasan S, Tsai CM, Khoa MT, et al. A prospective, molecular epidemiology study of EGFR mutations in Asian patients with advanced non-small-cell lung cancer of adenocarcinoma histology (PIONEER). J Thorac Oncol. 2014;9(2):154-62. https://doi.org/10.1097/JTO.0000000000 000033.

13. Wang LY, Cui JJ, Guo AX, Yin JY. Clinical efficacy and safety of afatinib in the treatment of non-small-cell lung cancer in Chinese patients. Onco Target Ther. 2018;2018(11):529-38. https://doi. org/10.2147/OTT.S136579.

14. Gristina V, La Mantia M, Galvano A, Cutaia S, Barraco N, Castiglia M, et al. Non-small cell lung cancer harboring concurrent EGFR genomic alterations: a systematic review and critical appraisal of the double dilemma. J Mol Pathol. 2021;2(2):173-96. 
15. Hirsh V. New developments in the treatment of advanced squamous cell lung cancer: focus on afatinib. Onco Targets Ther. 2017;2017(10):2513-26. https://doi.org/10.2147/OTT.S104177.

16. Solca F, Dahl G, Zoephel A, Bader G, Sanderson M, Klein C, et al. Target binding properties and cellular activity of afatinib (BIBW 2992), an irreversible ErbB family blocker. J Pharmacol Exp Ther. 2012;343(2):342-50. https://doi.org/10.1124/jpet.112. 197756.

17. Li D, Ambrogio L, Shimamura T, Kubo S, Takahashi M, Chirieac LR, et al. BIBW2992, an irreversible EGFR/HER2 inhibitor highly effective in preclinical lung cancer models. Oncogene. 2008;27(34):4702-11. https://doi.org/10.1038/onc.2008.109.

18. Park K, Tan EH, O’Byrne K, Zhang L, Boyer M, Mok T, et al. Afatinib versus gefitinib as first-line treatment of patients with EGFR mutation-positive non-small-cell lung cancer (LUX-Lung 7): a phase $2 \mathrm{~B}$, open-label, randomised controlled trial. Lancet Oncol. 2016;17(5):577-89. https://doi.org/10.1016/S14702045(16)30033-X.

19. Schuler M, Wu YL, Hirsh V, O’Byrne K, Yamamoto N, Mok $\mathrm{T}$, et al. First-line afatinib versus chemotherapy in patients with non-small cell lung cancer and common epidermal growth factor receptor gene mutations and brain metastases. J Thorac Oncol. 2016;11(3):380-90. https://doi.org/10.1016/j.jtho.2015.11.014.

20. Yang JC, Sequist LV, Zhou C, Schuler M, Geater SL, Mok T, et al. Effect of dose adjustment on the safety and efficacy of afatinib for EGFR mutation-positive lung adenocarcinoma: post hoc analyses of the randomized LUX-Lung 3 and 6 trials. Ann Oncol. 2016;27(11):2103-10. https://doi.org/10.1093/annonc/mdw322.

21. Hochmair MJ, Morabito A, Hao D, Yang CT, Soo RA, Yang $\mathrm{JC}$, et al. Sequential treatment with afatinib and osimertinib in patients with EGFR mutation-positive non-small-cell lung cancer: an observational study. Future Oncol. 2018;14(27):2861-74. https://doi.org/10.2217/fon-2018-0711.

22. Wu YL, Cheng Y, Zhou X, Lee KH, Nakagawa K, Niho S, et al. Dacomitinib versus gefitinib as first-line treatment for patients with EGFR-mutation-positive non-small-cell lung cancer (ARCHER 1050): a randomised, open-label, phase 3 trial. Lancet Oncol. 2017;18(11):1454-66. https://doi.org/10.1016/S14702045(17)30608-3.

23. Wu Y, Tu H, Feng J, Shi M, Zhao J, Wang Y, et al. P3.01-036. A phase IIIb open-label, single-arm study of afatinib in EGFR TKI-naïve patients with EGFRm+ NSCLC: an interim analysis. J Thorac Oncol. 2017;12(11):S2214. https://doi.org/10.1016/j.jtho. 2017.09.1477.

24. Halmos B, Tan EH, Soo RA, Cadranel J, Lee MK, Foucher P, et al. Impact of afatinib dose modification on safety and effectiveness in patients with EGFR mutation-positive advanced NSCLC: results from a global real-world study (RealGiDo). Lung Cancer. 2019;2019(127):103-11. https://doi.org/10.1016/j.lungcan.2018. 10.028 .

25. Kim ES, Halmos B, Kohut IF, Patel T, Rostorfer RD, Spira AI, et al. Efficacy and safety results of the afatinib expanded access program. Oncol Ther. 2017;5(1):103-10. https://doi.org/10.1007/ s40487-017-0043-5.

26. Cappuzzo F, Soo R, Hochmair M, Schuler M, Lam KC, Stehle G, et al. Global named patient use program of afatinib in advanced non-small-cell lung carcinoma patients who progressed following prior therapies. Future Oncol. 2018;14(15):1477-86. https://doi. org/10.2217/fon-2017-0666.

27. Thongprasert S, Geater S, Clement D, Abdelaziz A, ReyerIgama J, Jovanovic D, et al. 157P Second-line afatinib for patients with locally advanced or metastatic NSCLC harbouring common EGFR mutations: a phase IV study. J Thorac Oncol. 2018;13(4):S94. https://doi.org/10.1016/S1556-0864(18)30431-3.
28. Wang S, Xing P, Yang K, Hao X, Ma D, Mu Y, et al. Efficacy and safety of afatinib in a Chinese population with advanced lung adenocarcinoma with sensitive EGFR mutations. Thorac Cancer. 2019;10(6):1461-8. https://doi.org/10.1111/1759-7714.13095.

29. Li Y, Wang X, Wang L, Kou Y, Ma X, Feng JF, et al. Afatinib in the treatment of advanced NSCLC with EGFR mutation: an observational real-world study. J Clin Oncol. 2019;37(Suppl15): e20518. https://doi.org/10.1200/JCO.2019.37.15_suppl.e20518.

30. Liang SK, Hsieh MS, Lee MR, Keng LT, Ko JC, Shih JY. Realworld experience of afatinib as a first-line therapy for advanced EGFR mutation-positive lung adenocarcinoma. Oncotarget. 2017;8(52):90430-43. https://doi.org/10.18632/oncotarget.19563.

31. Lin YT, Chen JS, Liao WY, Ho CC, Hsu CL, Yang CY, et al. Clinical outcomes and secondary epidermal growth factor receptor (EGFR) T790M mutation among first-line gefitinib, erlotinib and afatinib-treated non-small cell lung cancer patients with activating EGFR mutations. Int J Cancer. 2019;144(11):2887-96. https://doi. org/10.1002/ijc.32025.

32. Tu CY, Chen CM, Liao WC, Wu BR, Chen CY, Chen WC, et al. Comparison of the effects of the three major tyrosine kinase inhibitors as first-line therapy for non-small-cell lung cancer harboring epidermal growth factor receptor mutations. Oncotarget. 2018;9(36):24237-47. https://doi.org/10.18632/oncotarget.24386.

33. Yoon SH, Kim YS, Chung JH, Seol HY, Kim MH. A realworld experience of first-line afatinib in Korean patients with EGFR-mutant non-small cell lung cancer. Ann Oncol. 2019;30(Supp19):ix163. https://doi.org/10.1093/annonc/mdz437. 011.

34. Soria J-C, Ohe Y, Vansteenkiste J, Reungwetwattana T, Chewaskulyong B, Lee KH, et al. Osimertinib in untreated EGFRmutated advanced non-small-cell lung cancer. N Engl J Med. 2017;378(2):113-25. https://doi.org/10.1056/NEJMoa1713137.

35. Ramalingam SS, Vansteenkiste J, Planchard D, Cho BC, Gray JE, Ohe Y, et al. Overall survival with osimertinib in untreated, EGFR-mutated advanced NSCLC. New Engl J Med. 2019;382(1):41-50. https://doi.org/10.1056/NEJMoa1913662.

36. Gristina V, Malapelle U, Galvano A, Pisapia P, Pepe F, Rolfo C, et al. The significance of epidermal growth factor receptor uncommon mutations in non-small cell lung cancer: a systematic review and critical appraisal. Cancer Treat Rev. 2020;85:101994. https:// doi.org/10.1016/j.ctrv.2020.101994.

37. Tanaka I, Morise M, Kodama Y, Matsui A, Ozawa N, Ozone S, et al. Potential for afatinib as an optimal treatment for advanced non-small cell lung carcinoma in patients with uncommon $E G F R$ mutations. Lung Cancer. 2019;127:169-71. https://doi.org/10. 1016/j.lungcan.2018.11.018.

38. Yang JCH, Schuler M, Popat S, Miura S, Heeke S, Park K, et al. Afatinib for the treatment of NSCLC harboring uncommon EGFR mutations: a database of 693 cases. J Thorac Oncol. 2020;15(5):803-15. https://doi.org/10.1016/j.jtho.2019.12.126.

39. Ito K, Morise M, Wakuda K, Hataji O, Shimokawaji T, Takahashi $\mathrm{K}$, et al. A multicenter cohort study of osimertinib compared with afatinib as first-line treatment for EGFR-mutated non-small-cell lung cancer from practical dataset: CJLSG1903. ESMO Open. 2021;6(3):100115. https://doi.org/10.1016/j.esmoop. 2021. 100115.

40. Hochmair MJ, Morabito A, Hao D, Yang CT, Soo RA, Yang JCH, et al. Sequential afatinib and osimertinib in patients with EGFR mutation-positive non-small-cell lung cancer: final analysis of the GioTag study. Future Oncol. 2020;16(34):2799-808. https://doi. org/10.2217/fon-2020-0740.

41. Leonetti A, Sharma S, Minari R, Perego P, Giovannetti E, Tiseo M. Resistance mechanisms to osimertinib in EGFR-mutated nonsmall cell lung cancer. Brit J Cancer. 2019;121(9):725-37. https:// doi.org/10.1038/s41416-019-0573-8. 


\section{Authors and Affiliations}

\section{Hai-Yan Tu ${ }^{1}$. Jifeng Feng ${ }^{2} \cdot$ Meiqi Shi $^{2}$. Jun Zhao ${ }^{3}$-Yuyan Wang ${ }^{3}$. Jianhua Chang ${ }^{4}$. Jialei Wang ${ }^{4}$. Ying Cheng ${ }^{5}$. Jing Zhu ${ }^{5}$. Eng-Huat Tan ${ }^{6} \cdot \mathrm{Kai} \mathrm{Li}^{7} \cdot$ Yiping Zhang ${ }^{8} \cdot$ Victor Lee $^{9,10}$. Cheng-Ta Yang ${ }^{11}$. Wu-Chou Su ${ }^{12}$. David Chi-Leung Lam ${ }^{13}$. B. J. Srinivasa ${ }^{14}$. Senthil Rajappa ${ }^{15}$. Ching-Liang Ho ${ }^{16} \cdot$ Kwok Chi Lam $^{17}$.

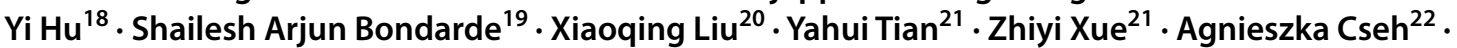 Dennis Chin-Lun Huang ${ }^{23}$. Caicun $\mathrm{Zhou}^{24}$ - Yi-Long Wu ${ }^{1}$ (])}

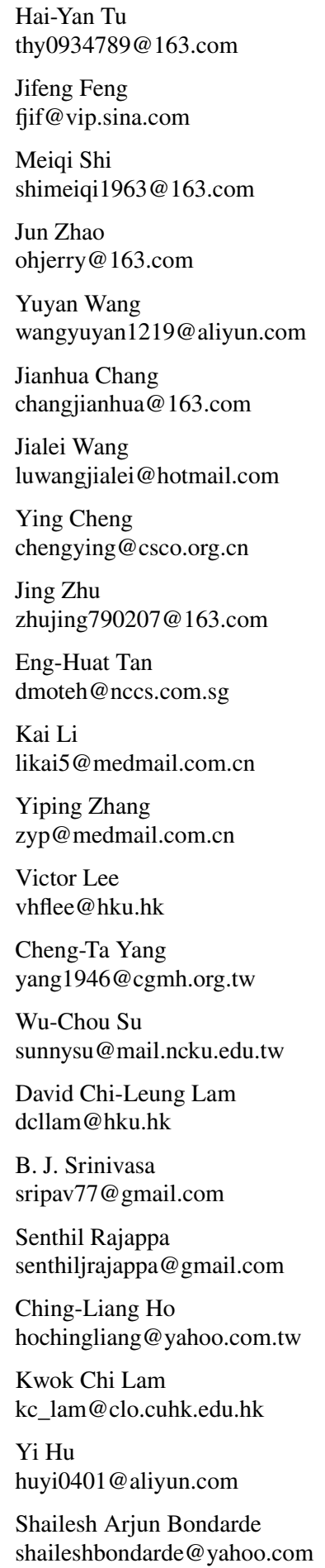

Xiaoqing Liu

liuxiaoqing@csco.org.cn

Yahui Tian

yahui.tian@boehringer-ingelheim.com

Zhiyi Xue

nick.xue@boehringer-ingelheim.com

Agnieszka Cseh

agnieszka.cseh@boehringer-ingelheim.com

Dennis Chin-Lun Huang

huangchinlun@gmail.com

Caicun Zhou

caicunzhoudr@163.com

1 Guangdong Lung Cancer Institute, Guangdong Provincial People's Hospital and Guangdong Academy of Medical Sciences, Guangzhou 510080, China

2 Department of Medical Oncology, Jiangsu Cancer Hospital and Jiangsu Institute of Cancer Research and The Affiliated Cancer Hospital of Nanjing Medical University, Nanjing 210029, China

3 Key Laboratory of Carcinogenesis and Translational Research (Ministry of Education/Beijing), Department 1 of Thoracic Oncology Medicine, Peking University Cancer Hospital and Institute, Beijing 100142, China

4 Fudan University Shanghai Cancer Center, Shanghai 200032, China

5 Division of Thoracic Oncology, Jilin Province Cancer Hospital, Changchun 130012, China

6 Department of Medical Oncology, National Cancer Centre, Singapore 169610, Singapore

7 Tianjin Medical University Cancer Institute and Hospital, Tianjin 300060, China

8 Zhejiang Cancer Hospital, Hangzhou 310022, China

9 Department of Clinical Oncology, Queen Mary Hospital, The University of Hong Kong, Hong Kong, China

10 Clinical Oncology Center, The University of Hong Kong-Shenzhen Hospital, Shenzhen 518053, China

11 Department of Thoracic Medicine, Linkou Chang Gung Memorial Hospital, Taoyuan 333, Taiwan

12 National Cheng Kung University Hospital, Tainan 704, Taiwan

13 Departments of Clinical Oncology, and Medicine, Queen Mary Hospital, The University of Hong Kong, Hong Kong, China

14 HCG Hospital, Bangalore 560020, India 
15 Basavatarakam Indo-American Cancer Hospital and Research Institute, Hyderabad 500034, India

16 Division of Hematology/Oncology, Tri-Service General Hospital, National Defense Medical Center, Taipei 114, Taiwan

17 Prince of Wales Hospital, Shatin, New Territories, Hong Kong, China

18 Department of Oncology, Chinese PLA General Hospital, Beijing 100853, China
19 Shatabdi Superspeciality Hospital, Nashik 422005, India

20 307th Hospital of PLA, Beijing 100070, China

21 Boehringer Ingelheim (China) Investment Co., Ltd, Shanghai, China

22 Boehringer Ingelheim International GmbH, 55216 Ingelheim, Germany

23 Boehringer Ingelheim Taiwan Limited, Taipei 104, Taiwan

24 Shanghai Pulmonary Hospital, Tongji University, Shanghai 200433, China 